\title{
Generation of a humanized mouse model with both human immune system and liver cells to model hepatitis $\mathbf{C}$ virus infection and liver immunopathogenesis
}

\author{
Moses T Bility ${ }^{1}$, Liguo Zhang ${ }^{2}$, Michael L Washburn ${ }^{3}$, T Anthony Curtis ${ }^{1}$, Grigoriy I \\ Kovalev ${ }^{1}$, and Lishan Su ${ }^{1,2}$ \\ ${ }^{1}$ Lineberger Comprehensive Cancer Center, University of North Carolina (UNC) at Chapel Hill, \\ Chapel Hill, North Carolina, USA \\ ${ }^{2}$ Center for Infection and Immunity, Institute of Biophysics, Chinese Academy of Sciences, \\ Beijing, China \\ ${ }^{3}$ GlaxoSmithKline, Research Triangle Park, North Carolina, USA
}

\section{Abstract}

Establishing a small animal model that accurately recapitulates hepatotropic pathogens, including hepatitis $\mathrm{C}$ virus (HCV) infection and immunopathogenesis, is essential for the study of hepatitis virus-induced liver disease and for therapeutics development. This protocol describes our recently developed humanized mouse model for studying HCV and other hepatotropic infections, human immune response and hepatitis and liver fibrosis. The first 5-h stage is the isolation of human liver progenitor and hematopoietic stem cells from fetal liver. Next, AFC8 immunodeficient mice are transplanted with the isolated progenitor/stem cells. This generally takes $2 \mathrm{~h}$. The transplanted mice are then treated for a month with the mouse liver apoptosis-inducing AFC8 dimerizer and left for an additional 2-month period to permit human liver and immune cell growth as well as system reconstitution and development before inoculation with $\mathrm{HCV}$ clinical isolates. HCV infection, human immune response and liver disease are observed with high incidence from approximately 2 months after inoculation.

\section{INTRODUCTION}

Several human pathogens including HCV, HBV (hepatitis B virus) and HIV have a narrow host species restriction (i.e., to humans and chimpanzees). To overcome host species restriction barriers to studying these pathogens in small animal models, several humanmouse chimeric models containing hemato-lymphoid or liver cells have been developed ${ }^{1-3}$.

\section{Development of human-mouse chimeric models for HCV}

The Alb-uPA/immunodeficient mouse models, which contain the mouse gene encoding the plasminogen activator, urokinase ( $P l a u$, referred to herein as $u P A$ ) under control of an

\footnotetext{
(C) 2012 Nature America, Inc. All rights reserved.

Correspondence should be addressed to M.T.B. (moses_bility@med.unc.edu) or L.S. (1su@ med.unc.edu).

AUTHOR CONTRIBUTIONS M.T.B. and L.S. organized and wrote the article; L.Z., M.L.W., T.A.C. and G.I.K. contributed to writing the article.

COMPETING FINANCIAL INTERESTS The authors declare competing financial interests: details are available in the online version of the paper.

Reprints and permissions information is available online at http://www.nature.com/reprints/index.html.
} 
albumin $(A l b)$ enhancer/promoter, allow for high human hepatocyte repopulation (90\%) in chimeric mice; however, homozygous animals are unhealthy and die because of profound hypofibrinogenemia and accelerated hepatocyte death, if not rescued by transplantation of mouse or human hepatocytes ${ }^{1,4,5} . A l b-u P A^{+/+}$immunodeficient mouse with high human adult hepatocyte engraftment can be infected with $\mathrm{HCV}$ or HBV ${ }^{1}$. In addition, the

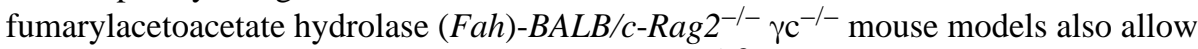
human hepatocyte engraftment and HCV infection ${ }^{6-8}$. However, these human-mouse chimeric liver mouse models lack a functional human immune system; thus, it is not possible to study viral-induced immunopathogenesis, and no virus-induced liver diseases are

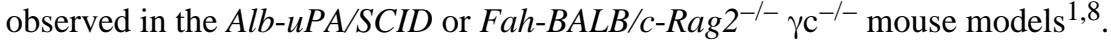

To overcome the problems associated with human-mouse chimeric liver mouse models, we developed a novel humanized mouse model (AFC8) with both a human immune system and

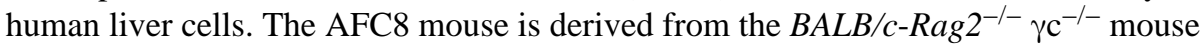
carrying a liver-specific suicidal transgene with inducible activity. The BALB/c-Rag $2^{-/-}$ $\gamma \mathrm{c}^{-I-}$ mouse, which lacks functional T, B and natural killer (NK) cells, supports the development of a functional human immune system after intrahepatic injection of $\mathrm{CD} 34^{+}$ human hematopoietic stem cells (HSCs) $)^{2,3,9}$. To facilitate engraftment and repopulation of human liver cells in $B A L B / c-R_{a g} 2^{-/-} \gamma \mathrm{c}^{-/}$mice, we expressed active Caspase 8 fused with FK506-binding domain (FKBP) under the control of the $A l b$ promoter to enable druginducible suicidal activity in mouse liver cells (AFC8 mice; Fig. 1) ${ }^{10}$. We added an Nterminal myristoylation signal $(\mathrm{M})$ to the transgene to promote membrane anchoring and added the simian virus 40 polyadenylation signal (SV40 PolyA) to improve RNA stability and translation (Fig. 1). We co-transplanted human liver/hepatic progenitor cells (HPCs) and CD34 ${ }^{+}$HSCs into the AFC8 transgenic mice and treated them with the FKBP dimerizer (AP20187; Fig. 2). The AFC8 mouse model allows the development of human liver and immune cells, thereby generating a mouse model, AFC8-hu HSC/Hep, containing both a human immune system (Fig. 3a) and hepatocytes (Fig. 3b) ${ }^{11}$. The AFC8-hu HSC/Hep mice supported $\mathrm{HCV}$ infection in the liver and generated a human T cell response to HCV. In addition, HCV infection induced liver inflammation and fibrosis, which correlated with the activation of human hepatic stellate cells and the expression of human fibrogenic genes ${ }^{11}$.

\section{Applications of the model}

The AFC8 model is the first humanized mouse model to incorporate a functional humanmouse chimera liver and immune system in the same animal, thus allowing the study of hepatitis virus-induced liver immunopathogenesis (Figs. 3 and 4). HCV infection in the AFC8 model results in increased numbers of human leukocytes $\left(\mathrm{CD}_{4} 5^{+}, \mathrm{CD}^{+}\right.$and $\mathrm{CD} 4^{+}$, plasmacytoid dendritic cells, macrophages and T regulatory cells) in the liver and anti-HCV $\mathrm{T}$ cell immune response ${ }^{11}$. HCV infection in the AFC8 model is also associated with elevated alanine aminotransferase (ALT) levels and liver fibrosis ${ }^{11}$. The AFC8 model is thus ideal for studying the role of various human immune cells in the induction and maintenance of virus-induced liver damage and fibrosis. In addition, the AFC8 model is useful for evaluating antiviral drugs and immunotherapeutics (vaccines, neutralizing antibodies and so on). In addition to studying HCV infection and liver immunopathology, the AFC8 model could potentially be used to study liver immunopathogenesis induced by other hepatotropic pathogens including HBV, HDV (hepatitis D virus) and so on. Current small-animal liver fibrosis models use a chemical agent (carbon tetrachloride, diethylnitrosamine and so on) or surgical procedure (bile duct ligation); however, these models do not accurately mimic hepatitis and associated liver fibrosis in human patients, which are predominately caused by hepatitis virus infections ${ }^{12}$. The AFC8 model is the first model to recapitulate human hepatitis virus-induced liver fibrosis, thus providing a robust platform for preclinical evaluation of antifibrotic therapeutics. The widespread use of antiretroviral drugs has 
markedly reduced HIV-associated mortality; however, HIV and hepatitis virus co-infection, and its associated 'enhanced' liver disease, has emerged as a major mortality factor owing to shared transmission routes ${ }^{13,14}$. The AFC8 model is the only small-animal model capable of supporting HCV and HIV co-infection.

Comparison with other models-The AFC 8 humanized mouse model uses a caspase 8-dependent induction of mouse hepatocyte apoptosis to promote human hepatocyte repopulation following co-transplantation of fetal liver/HPCs and HSCs. This method of mouse hepatocyte suicide is much less toxic compared with the UPA, FAH or hepatotoxicant (CCl4) model ${ }^{11,15,16}$. The 'toxic' models cause substantial liver damage during the promotion of human hepatocyte repopulation, thus making it impossible to study viral-induced liver diseases. On the contrary, the AFC8 model does not have background toxicity or liver disease because of the tissue-specific and tightly regulated mechanism of apoptosis induction in mouse hepatocytes (Fig. 1). This tightly regulated liver damage model could explain the higher repopulation potential of the AFC8 mice with fetal liverderived cells as compared with the Alb-uPA or Fah mice ${ }^{11,17}$. The highly 'toxic' uPA and FAH mouse model liver environment, although highly permissive for adult liver-derived hepatocyte repopulation, could be inhospitable to fetal liver-derived cell repopulation.

In addition, the AFC 8 model is the only humanized mouse model that enables hepatitis virus-induced liver immunopathology from the co-development of human liver and immune cells in the model ${ }^{11}$. Current human-mouse chimera mouse models do not have human immune cells; thus, the role of the human immune system in controlling infection or promoting disease cannot be examined ${ }^{16}$. The biggest disadvantage of the AFC8 humanized (liver and immune system) model is the level of human hepatocyte repopulation, which is approximately $15-25 \%$ compared with the uPA or FAH model that readily achieves human hepatocyte repopulation upward of $80 \%$ (ref. 11). However, it should be noted that the uPA or FAH models only show high human hepatocyte repopulation with human adult liverderived cells but not with fetal liver-derived cells ${ }^{17}$. Transplantation of AFC 8 mice with human adult liver-derived hepatocytes results in relatively higher human hepatocyte repopulation levels compared with fetal liver-derived cells (data not shown). The low hepatocyte repopulation with fetal liver-derived cells could partly explain why the virus genome is readily detected in the liver of infected mice but not readily detected in the serum of infected mice. In addition, the transplantation of fetal liver cells as opposed to adult differentiated hepatocytes in the UPA or FAH model results in a less mature human liver ${ }^{11}$. Differences in differentiation state between fetal and adult-derived hepatocytes could also explain the lack of serum HCV genome, as adult hepatocytes could more efficiently support higher viral replication and particle release in chimeric animals ${ }^{15,16}$.

\section{Experimental design}

The AFC8 mouse was developed on the BALB/c-Rag2 ${ }^{-/-} \mathrm{\gamma c}^{-/-}$mouse background according to protocols and methods approved by the Institutional Animal Care and Use Committee (IACUC). Previous work by our laboratory and others has shown robust human

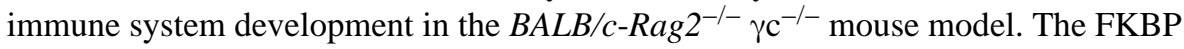
construct was developed by ARIAD Pharmaceuticals and shows robust control of regulated target protein in a ligand-dependent manner (FKBP dimerizer-AP20187) ${ }^{18}$. Caspase 8 protein is activated by the formation of a dimer complex, and thus the FKBP was the ideal system for ligand-inducible activation, as this method has been used with great efficiency for similar purposes. The human gene encoding caspase 8 was inserted under the regulatory control of the FKP ligand-binding activation domain; this cassette was in turn placed under the transcriptional control of the mouse Alb promoter, including enhancer elements (Fig. 1). The resulting transgene construct has been robustly tested using cell culture methodologies. 
Optimal tissue-specific and ligand-regulated constructs were used to construct transgenic mice. The early liver (15 to 18 weeks gestation) is an active organ for hematopoiesis, thus providing a convenient source of both HSCs and progenitor liver cells. The human CD34 ${ }^{+}$ marker is a pan-human HSC marker that has been widely used in HSC isolation and selection ${ }^{19}$. The human fetal liver cells include hepatoblasts and stromal cells, thus providing hepatitis virus target cells and supporting cells (hepatic stellate cells and endothelial cells) that have crucial roles in fibrogenesis ${ }^{11}$. Intrahepatic transplantation is recommended because it allows rapid and consistent transplantation of a large cohort of mice $^{11}$. The animals should be irradiated before transplantation to eliminate residual mouse immune cells and hematopoietic lineage progenitor/stem cells and also to promote human cell engraftment and regeneration ${ }^{11,19}$. Human liver repopulation is promoted via the activation of the AFC8 model's transgene. Humanized mice should be inoculated with hightiter HCV patient serum to ensure infection; PBS and virus-inactivated or uninfected serum should be used as controls. Untransplanted mice inoculated with patient serum can be used as controls to demonstrate that human cells are necessary for infection and liver pathology. In addition, during model development, mice transplanted with human adult hepatocytes were inoculated with patient serum to demonstrate that HSC-derived human immune system was necessary for anti-HCV immune response and liver immunopathology ${ }^{11}$.

\section{Limitations}

This model is, to our knowledge, the first and only small-animal model with both a human immune system and human liver cells for studying hepatitis virus infection and associated liver pathology. This model, however, has several limitations. The relatively low level of repopulation and the immature phenotype of the human hepatocytes are probably responsible for the very low serum levels of human albumin and $\mathrm{HCV}$ viremia ${ }^{11}$. This limits the use of the AFC8 model for longitudinal analysis of anti-HCV therapeutics. Furthermore, the immature hepatocyte differentiation may hinder the use of the model for examining human-specific xenobiotic metabolism and associated toxicity. Finally, similarly to all current mouse models with humanized immune system, the antiviral immune response may not be as robust as in human patients. Although HCV-reactive T cell response can be detected, the B cell response is relatively weak, with predominant IgM response and relatively low $\mathrm{IgG}$ response. Future studies addressing these deficiencies are needed in order to develop a fully robust preclinical model for studying hepatotropic pathogens, human immune response and drug metabolism and liver toxicity.

\section{MATERIALS}

\section{REAGENTS}

- Human HCV genotype 1A clinical isolates. These should be obtained from HCVpositive patients with $>1 \times 10^{8}$ genome copies per $\mathrm{ml}$ serum viral load. For negative control serum from the same patient, heat-inactivate $\mathrm{HCV}$-positive serum at $65{ }^{\circ} \mathrm{C}$ for $4 \mathrm{~h}$. Human mock serum should be obtained from healthy (HCV-, HBV-, HIV-negative) individuals ! CAUTION Patient sample collection must conform to governmental and institutional guidelines, and informed consent must be obtained from all human subjects or donors. Serum samples are infectious and should be handled using Biosafety level (BSL) $2^{+} / 3$-recommended protocols.

- Human fetal liver, 15- to 18-week gestation period (Advanced Bioscience Resources) ! CAUTION Human tissues should be handled using BSL2recommended protocols and should be collected and used in accordance with all institutional and governmental ethics guidelines. 
- Newborn AFC8 mice ( 2 to $5 \mathrm{~d}$ old; BALB/c-Rag $2^{-/-} \gamma \mathrm{c}^{-/}$mice, expressing active Caspase 8 fused with FK506-binding domain under control of the Alb promoter) ! CAUTION All experiments using live rodents must conform to governmental and institutional guidelines.

- Sulfamethoxazole-trimethoprim (SMZ; Hi-Tech Pharmacal, cat. no. 0823-16)

- AP20187 (induces FKBP/caspase 8 dimerization and activation, mouse liver apoptosis and human liver repopulation; Ariad Pharmaceuticals)

- $\quad$ Liver digestion medium (Gibco, cat. no. 17703)

- Ficoll (GE Healthcare, cat. no. 17-1440-03)

- Human CD34 indirect microbead kit (Miltenyi Biotec, cat. no. 130-046-702)

- Running buffer (autoMACS running buffer, Miltenyi Biotec, cat. no. 130-091-221; alternatively, use a $2 \mathrm{mM}$ EDTA, $0.5 \%$ (wt/vol) BSA/PBS solution)

- Rinsing solution (autoMACS rinsing solution, Miltenyi Biotec, cat. no. 130-091-222; alternatively, use 2 mM EDTA/PBS solution)

- Iscove's modified Dulbecco's medium (IMDM; Invitrogen, cat. no. 12440-046)

- Fetal bovine serum (FBS; Gibco, cat. no. 16000044)

- Penicillin-streptomycin (pen-strep; Gibco, cat. no. 1541)

- Red blood cell lysis buffer (1× ACK lysis buffer; Invitrogen, cat. no. A10492-01)

- L-Glutamine (Gibco, cat. no. 25030)

- DNase at $5 \mathrm{mg} \mathrm{ml}^{-1}$ (Roche, cat. no. 10104159001)

- Avertin (2,2,2-Tribromoethanol; Sigma-Aldrich, cat. no. T48402)

- Antibodies and dyes: human-specific CD34 (Clone AC136; Miltenyi Biotec, cat. no. 130-081-001), human-specific CD45 (Clone H130, BioLegend, cat. no. 304014; Clone 2B11 + PD7/26, Dako, cat. no. IR75161), mouse-specific CD45 (Clone 30-F11, BioLegend, cat. no. 103126), human-specific CD3 (Clone HIT3a, BD Bioscience, cat. no. 300306; Dako, cat. no. IR50361), human-specific CD123 (Clone 6H6, BioLegend, cat. no. 306008), human-specific CD11c (Clone Bu15, BioLegend, cat. no. 337208), human-specific CD19 (Clone HIB19, BioLegend, cat. no. 302210), human-specific CD14 (Clone M5E2, BD Bioscience, cat. no. 558121), human-specific CD68 (Clone KP1, Dako, cat. no. IR60961), humanspecific albumin (Dako, cat. no. F011702), human-specific hepatocyte (HepPar1, Clone OCH1E5, Dako, cat. no. IR62461), human-specific a-smooth muscle actin (SMA) (Clone 1A4, Dako, cat. no. IR61161), human-specific glial fibrillary acidic protein (GFAP) (Abcam, cat no. 24109), 7-aminoactinomycin D (7-AAD; Invitrogen, cat. no. A1310), LIVE/DEAD fixable yellow dead cell stain kit (Invitrogen, cat. no. L34959)

- Trypan blue (Invitrogen, cat. no. 15250-061)

- Human albumin ELISA kit (Bethyl)

- Liquid nitrogen

- RNAlater (Qiagen)

- Sirius Red/Fast Green or Masson's trichrome stain (Sigma-Aldrich) 
- AutoMACS cell separator (Miltenyi Biotec) or other manual/automated cell separators

- Hemocytometer

- Guava easyCyte mini flow cytometer (Millipore) and/or multicolor flow cytometer

- XRAD 320 biological irradiator (precision X-ray, CT) or other X-ray irradiators

- Microscope lamps

- 27-gauge needles (-0.5 $\mathrm{ml}$ insulin syringes)

- Heat lamp

- Operating scissors

- Scalpels

- Microcentrifuge tubes

- Aluminum foil

- Paper towels

\section{REAGENT SETUP}

Cell wash buffer-Freshly prepare using complete IMDM with $2.5 \mu 1$ of DNase added per $50 \mathrm{ml}$.

Antibody mix for CD34 + HSC yield and purity-Add $2 \mu 1$ of human-specific CD34 FITC, $2 \mu 1$ of human-specific CD45 phycoerythrin (PE) and $0.5 \mu 1$ of 7-AAD in $50 \mu 1$ of $2 \%$ (vol/vol) FBS/PBS solution per sample before staining.

Antibody mix for human immune repopulation-Add $2 \mu \mathrm{l}$ of human- specific CD45 FITC, $2 \mu 1$ of mouse-specific CD45 PE and $0.5 \mu 1$ 7-AAD in $50 \mu \mathrm{l}$ of $2 \%$ (vol/vol) FBS/PBS solution per sample before staining.

Medium-Freshly prepare using IMDM supplemented with 10\% (vol/vol) FBS, $100 \mathrm{U}$ $\mathrm{ml}^{-1}$ penicillin and $100 \mu \mathrm{g} \mathrm{ml}^{-1}$ streptomycin, and $2 \mathrm{mM} \mathrm{L-glutamine.}$

\section{PROCEDURE}

\section{Liver digestion • TIMING $1 \mathrm{~h}$}

1) Warm up the wash buffer in a $37^{\circ} \mathrm{C}$ water bath.

2| Add $2.5 \mu 1$ of DNase for every $50 \mathrm{ml}$ of liver digestion medium (this creates the liver digestion solution). Prepare $100 \mathrm{ml}$ per liver to be digested.

3| Pour a single 15- to 18-week gestation period human fetal liver into a 10-cm dish; use scalpels to separate the connective tissue from the parenchyma.

4) Discard the connective tissue and chop the parenchymal tissue into small pieces $\left(1-2 \mathrm{~mm}^{3}\right)$. Alternatively, the tissue can be dissociated using automated tissue dissociators (for example, Gentle MACS, Miltenyi Biotec) according to the manufacturer's recommended protocol.

5| Transfer the tissue to a 50-ml tube with a $25-\mathrm{ml}$ pipette; wash the dish with 10 $\mathrm{ml}$ of liver digestion solution and add it to the same tube. 
6| Fill the tube with liver digestion solution to a 40-ml total volume.

7기 Wrap the tube cap with Parafilm.

$\triangle$ CRITICAL STEP This will prevent contamination of the sample.

8| Shake the tube for 5-10 s, place the tube in the $37^{\circ} \mathrm{C}$ water bath for $30 \mathrm{~min}$ and shake the tube every $5 \mathrm{~min}$.

9| The larger pieces will settle to the bottom; remove the liquid portion and filter it through a 70- $\mu \mathrm{m}$ filter into a new 50-ml tube; place the new tube on ice.

10| Add $35 \mathrm{ml}$ of liver digestion solution to the tube (from Step 9) containing the larger pieces of liver tissue and repeat Steps 8 and 9.

11 Spin the two tubes with liver cells at $252 \mathrm{~g}$ for $10 \mathrm{~min}$ at $4{ }^{\circ} \mathrm{C}$.

12 Decant the supernatant, resuspend the cells with $40 \mathrm{ml}$ of wash buffer and combine the tubes into one 50-ml tube.

\section{Liver and HPCs separation - TIMING $1 \mathrm{~h}$}

13 Spin the suspension down at $18 g$ at $4{ }^{\circ} \mathrm{C}$ for $5 \mathrm{~min}$.

14| Remove the supernatant and transfer to a new 50-ml tube and store on ice; this is for CD34+ HSC separation.

15| Resuspend the pellet with $14 \mathrm{ml}$ of wash buffer and transfer it to a 15-ml conical tube.

16 Spin down the suspension from Step 15 at $18 g$ for 5 min at $4{ }^{\circ} \mathrm{C}$ and transfer the supernatant to a new tube on ice; this will be combined with the supernatant from Step 14 for HSC purification.

17| Resuspend the pellet from Step 16 with $14 \mathrm{ml}$ of wash buffer and repeat Step 16.

The pellet should appear white or yellow (not red) in color. The light-colored pellet contains liver progenitor cells.

\section{? TROUBLESHOOTING}

18 Resuspend the final pellet containing liver progenitor cells from Step 17 with 10 $\mathrm{ml}$ of wash buffer and transfer in a new 15-ml tube (labeled with the tissue identification number and liver progenitor cells); store the tube on ice until Step 50. Remove $20 \mu \mathrm{l}$ and place in a 1.5-ml microcentrifuge tube. Add $180 \mu \mathrm{l}$ of wash buffer to dilute. Count cells using a hemocytometer after trypan blue staining (mix $10 \mu \mathrm{l}$ of cells with $10 \mu \mathrm{l}$ of trypan blue and count clear cells as live cells).

\section{Lymphocyte purification • TIMING $1 \mathrm{~h}$}

19| Spin down the collected supernatants containing CD34 ${ }^{+}$HSCs from Steps 14,16 and 17 at $469 \mathrm{~g}$ for $5 \mathrm{~min}$ at $4^{\circ} \mathrm{C}$, combine the pellets and resuspend in a total volume of $35 \mathrm{ml}$ of wash buffer.

20| Fill a 10-ml pipette with $14 \mathrm{ml}$ of Ficoll and eject $11 \mathrm{ml}$ very slowly at the bottom of the tube under the cell suspension.

$\triangle$ CRITICAL STEP Eject the Ficoll with a slow, constant flow, being extremely careful not to disturb the interface. Retain $3 \mathrm{ml}$ of Ficoll in the pipette to prevent air bubbles. 
21 Carefully move the tube to the centrifuge, and then spin the suspension down at $1,880 \mathrm{~g}$ for $30 \mathrm{~min}$ at room temperature $\left(24^{\circ} \mathrm{C}\right)$.

22 Take a 10-ml pipette and remove the cells at the interface, trying to remove as little of the Ficoll as possible.

23 Transfer the collected cells into a 50-ml tube and add wash buffer up to $40 \mathrm{ml}$.

24| Remove $20 \mu \mathrm{l}$ and add it to $180 \mu \mathrm{l}$ of wash buffer in a 1.5 -ml tube (labeled 'ori10x dilution') and place the tube on ice. Count the cells using a hemocytometer and trypan blue dye. You should recover approximately $5 \times 10^{8}$ cells from each tissue.

\section{CD34 ${ }^{+}$cell purification $\bullet$ TIMING $2 \mathrm{~h}$}

25| Spin the suspension at $469 \mathrm{~g}$ at $4{ }^{\circ} \mathrm{C}$ for $5 \mathrm{~min}$ and discard the supernatant and resuspend the pellet in $10 \mathrm{ml}$ of wash buffer.

26| Spin the suspension at $469 \mathrm{~g}$ at $4{ }^{\circ} \mathrm{C}$ for $5 \mathrm{~min}$ and discard as much of the supernatant as possible without disturbing the pellet.

27| Resuspend the cells in $400 \mu \mathrm{l}$ of wash buffer for every $1 \times 10^{8}$ cells counted by trypan blue in Step 24.

28| Add $100 \mu 1$ of the FcR blocking reagent (in the human CD34 indirect microbead kit) for every $1 \times 10^{8}$ cells counted in Step 24 and mix well.

29| Add $100 \mu 1$ of the CD34-hapten-antibody reagent (in the human CD34 indirect microbead kit) for every $1 \times 10^{8}$ cells counted in Step 24 and mix well.

30| Incubate on ice for $30 \mathrm{~min}$.

31 Add $40 \mathrm{ml}$ of wash buffer to the cells.

32 Spin down the cell suspension at $469 \mathrm{~g}$ for $5 \mathrm{~min}$ at $4{ }^{\circ} \mathrm{C}$ and discard the supernatant.

33| Resuspend the cells in $400 \mu \mathrm{l}$ of wash buffer for every $1 \times 10^{8}$ cells counted in Step 24.

34) Add $100 \mu 1$ of the Anti-Hapten MicroBeads reagent (in the human CD34 indirect microbead kit) for every $1 \times 10^{8}$ cells counted in Step 24; mix well and incubate on ice for $30 \mathrm{~min}$.

$\triangle$ CRITICAL STEP Failure to incubate for at least $30 \mathrm{~min}$ will result in lower $\mathrm{CD} 34^{+}$purity and yield.

35| Resuspend the cells with $40 \mathrm{ml}$ of wash buffer and spin down the cell suspension at $469 \mathrm{~g}$ for $5 \mathrm{~min}$ at $4{ }^{\circ} \mathrm{C}$ and remove the supernatant. Resuspend the pellet in $3 \mathrm{ml}$ of wash buffer and transfer it to a 15-ml tube (labeled with the tissue identification number and the protocol step number).

36| Repeat Step 35 and transfer the residual cells into the same 15-ml tube (there should be a volume of $6 \mathrm{ml}$ in this tube); put the tube on ice.

37| Check the autoMACS cell separator machine to ensure that the run buffer and rinse buffer containers are filled (full) and the waste bottle is empty (not full).

38 Turn on the autoMACS cell separator machine and run the cleaning program.

39| Run the cell separation program using the cell suspension from Step 36, ensuring that the cells are well mixed. 
40| Rinse between samples if there is more than one.

41 When finished, count the number of cells; if the positive fraction is lower than expected, re-run the negative fraction until desirable separation is obtained.

42| Run the cleaning program and clean the machine.

43 Combine positive fractions (CD34+ HSCs; if more than one) and label the tubes with the tissue identification number.

44| Spin down the negative and positive fractions separately at $469 g$ at $4{ }^{\circ} \mathrm{C}$ for 5 $\min$.

45| Resuspend each fraction in $10 \mathrm{ml}$ of wash buffer and store on ice.

46| Mix each fraction; take $20 \mu \mathrm{l}$ from each fraction and add $180 \mu \mathrm{l}$ of wash buffer to each (label the tubes 'pos10x dilution' and 'neg10x dilution,' respectively).

47| Place the 'pos $10 \times$ dilution' and 'neg10 $\times$ dilution' tubes on ice. Note that you should have two 15-ml tubes containing 10-ml cell suspensions on ice, one labeled pos, one labeled neg, and three 1.5-ml tubes labeled 'ori10× dilution', 'pos10x dilution' and 'neg $10 \times$ dilution'.

48 Add $180 \mu \mathrm{l}$ of wash buffer to two 1.5-ml tubes labeled 'ori100× dilution' and 'neg100× dilution'. Transfer $20 \mu$ from tubes labeled 'ori $10 \times$ dilution' and 'neg $10 \times$ dilution' (ensure that the tubes are mixed thoroughly) to their respective $100 \times$ dilution tubes. Store all tubes back on ice. Determine CD $34^{+}$cell purity and number by staining the cells in tubes labeled 'ori100x dilution', 'pos $10 \times$ dilution' and 'neg100× dilution' for $\mathrm{CD}^{4} 4^{+}, \mathrm{CD} 45^{+}$and $7-\mathrm{AAD}^{+}$(dead) cells using an integrated flow cytometry - cell counter system or a combination of multicolor flow cytometry and the hemocytometer/trypan blue counting method. Use human $\mathrm{CD}_{3} 4^{+} \mathrm{HSC}$ or a human $\mathrm{CD}^{+} 4^{+}$cell line and isotype antibodies as controls.

\section{Cell preparation for injection • TIMING 30-40 min}

49| Spin down the CD34+ HSCs obtained from Step 45 at $469 g$ for 5 min at $4{ }^{\circ} \mathrm{C}$ and resuspend them with wash buffer to $1 \times 10^{6} \mathrm{ml}^{-1}$.

PAUSE POINT CD34+ HSCs can be stored overnight on ice for next-day transplantation if desired.

50| Spin down the liver/HPCs from Step 18 at $18 g$ for 5 min at $4{ }^{\circ} \mathrm{C}$ and resuspend them with wash buffer to $1 \times 10^{6} \mathrm{ml}^{-1}$.

PAUSE POINT At this point, liver progenitor cells can be stored overnight on ice for next-day transplantation if desired.

51 Prior to transplantation, spin down CD34+ HSCs (from Step 49) at $469 g$ and HPCs (from Step 50) at $18 \mathrm{~g}$ for $5 \mathrm{~min}$ at $4{ }^{\circ} \mathrm{C}$ and resuspend each cell type with wash buffer to 0.5 to $1 \times 10^{6} / 17.5 \mu \mathrm{l}$. Mix approximately 0.5 to $1 \times 10^{6} \mathrm{HSCs}$ and 0.5 to $1 \times 10^{6}$ liver progenitor cells $(35 \mu$ l total volume) for each mouse to be injected.

$\triangle$ CRITICAL STEP Cell suspension must be prepared at an approximately 35$\mu l$ volume per mouse, as injecting animals above this volume will result in animal death or transplantation failure. 


\section{Transplantation of human cells • TIMING $1.5 \mathrm{~h}$}

52| Take the cells from Step 51 to the animal room.

53| Irradiate 2- to 5-d-old newborn AFC8 mice in a sterilized paper bag with a single dose of $200 \mathrm{rad}$ using Xrad or other irradiators.

$\triangle$ CRITICAL STEP Mice less than $2 \mathrm{~d}$ old have lower survivability and mice older than $5 \mathrm{~d}$ old are difficult to transplant because it is difficult to see the liver through the skin.

54 Prepare an ice bucket wrapped with autoclaved aluminum foil in the hood.

$\triangle$ CRITICAL STEP Be careful when handling the foil so that the side the pups will be in contact with is not contaminated by unsterilized surfaces.

55| Set up the warming light in the hood and put a cage lid covered with sterile paper towels under it.

$\triangle$ CRITICAL STEP This is for mouse recovery after cold anesthesia and injection.

56 Put a clean cage, as well as the cage the pups are in, in the hood; separate the mother from the pups, move the pups to the clean cage and move the mother back to the housing rack.

57| Place one pup on the foil in the ice bucket and let the animal cool for about 1 min.

$\triangle$ CRITICAL STEP This is for light anesthesia.

58 Resuspend the cells immediately prior to injection every time. Load the syringe (27-gauge needles, $-0.5 \mathrm{ml}$ insulin syringes) with the cell suspension at a volume of $30 \mu \mathrm{l}$ per mouse.

59| Remove the first pup and place the next on ice (the actual injection step takes only a few seconds and the other pup can be cooled down by the time you are finished with the first one).

60| Restrain the pup by placing your index finger between its front legs (slightly cranially to where you would expect the collarbone to be) and your thumb between its hind legs and applying gentle pressure down and outward (just enough to pull the skin a little tight without injuring the animal).

61 Begin by vertically positioning the needle slightly to the left of the right lobe and angled slightly toward the right lobe; push the needle in about halfway, and then deposit the cells into the right lobe at the rate of $2 \mathrm{~s}$ per injection volume. The liver should be visible as a dark area located cranially to the stomach; it should be the largest organ in young pups. The stomach is identifiable by a white color on the animal's heart side. Release the pressure immediately with your fingers. Thereafter, remove the needle, being careful to avoid contact with your finger.

62 Repeat Steps 58-61 until the last mouse has been injected; place the pups back in their nest and return them to the mother. Perform similar procedures as stated above on control mice using vehicle (wash buffer without cells; nontransplanted AFC8 controls). Also use commercially available human primary hepatocytes and human HSCs as controls.

63 House mice in a pathogen-free facility and microisolator cages. Feed animals with autoclaved or irradiated food and maintain them on acidified autoclaved 
water with or without sulfamethoxazole-trimethoprim (SMZ) (7.8 ml of SMZ per $250 \mathrm{ml}$ of drinking water) on alternate weeks for the duration of the animals' lives.

$\triangle$ CRITICAL STEP Failure to keep mice under sterile conditions at all times will result in lower reconstitution and/or will increase opportunistic infections.

\section{Human liver and immune system reconstitution • TIMING 3 months}

64 Wean animals at 3 weeks of age and house at 5 humanized animals per cage.

65 Continue to feed animals with autoclaved/irradiated food and maintain them on acidified autoclaved water with or without SMZ (7.8 $\mathrm{ml}$ of SMZ per $250 \mathrm{ml}$ of drinking water) on alternate weeks for the duration of their lives.

66 Approximately 3-4 weeks after transplantation, administer an intraperitoneal injection of AP20187 (Ariad Pharmaceuticals) at a dose of $5 \mu \mathrm{g}$ per $\mathrm{g}$ body weight to AFC8/DKO-hu and control mice. Repeat the injection once a week for 4 weeks.

67| At 16 weeks after transplant, heat AFC8-hu HSC/Hep and control mice with a heat lamp and bleed them by making a single 5-mm cut (nick) on the tail vein with a sterile scalpel. Collect $100 \mu \mathrm{l}$ of blood per mouse in 1.5 -ml sterile microcentrifuge tubes containing $100 \mu \mathrm{l}$ of $20 \mathrm{mM}$ PBS-EDTA and place it on ice; samples can be kept on ice for up to $12 \mathrm{~h}$.

68 Spin collected blood-PBS/EDTA solution at $469 g$ for $10 \mathrm{~min}$ at $4{ }^{\circ} \mathrm{C}$ using a centrifuge. The top portion (plasma) can be used to examine human liver reconstitution. The bottom portion (peripheral blood mononuclear cells (PBMCs)) can be used to measure human immune reconstitution.

69| Resuspend the bottom portion (PBMCs) in red blood cell lysis buffer (1× ACK lysis buffer), incubate for $5 \mathrm{~min}$ at room temperature $\left(25^{\circ} \mathrm{C}\right)$, spin cells at $469 g$, repeat this procedure and resuspend in $2 \%$ (vol/vol) FBS/PBS containing human CD45, mouse CD45 and 7-AAD antibody mixture. Examine the human immune reconstitution (\% of human $\mathrm{CD}^{2} 5^{+}$cells/total $\mathrm{CD} 45^{+}$cells) using flow cytometry analysis.

\section{? TROUBLESHOOTING}

70| To measure human liver reconstitution, perform human albumin ELISA using plasma and the human albumin ELISA kit (Bethyl) according to the manufacturer's procedures.

\section{? TROUBLESHOOTING}

\section{Virus inoculation • TIMING $2 \mathrm{~h}$}

71 Administer a sublethal dose of Avertin (225-240 mg kg-1) via intraperitoneal injection to AFC8-hu HSC/Hep and control mice at 16 weeks after transplantation.

72| Inoculate anesthetized AFC8-hu HSC/Hep mice or control mice via retro-orbital injection with $75-100 \mu \mathrm{l}$ of human clinical isolates of HCV genotype 1a (1 to 5 $\times 10^{7}$ genome copies per ml) or control (PBS, uninfected patient serum, heatinactivated inoculum and so on) ${ }^{11}$. House and feed mice as described earlier. 


\section{HCV infection, liver disease and immune response • TIMING 2-5 months}

73| for longitudinal analysis of infection, liver disease and immune response, bleed the mice as described in Steps 67 and 68, approximately every 4 weeks following the initial bleeding at 2 weeks after inoculation.

74| Monitor the mice weekly and euthanize them at the chosen time point after inoculation. In our hands, HCV infection, human immune response, hepatitis and liver fibrosis were observed with high incidence 8 weeks after inoculation at the earliest. Animal survivability is markedly decreased 16 weeks after inoculation. Euthanize mice by administering $800 \mu \mathrm{l}$ of Avertin (lethal dose). Collect $500 \mu \mathrm{l}$ of blood (serum) and $500 \mu \mathrm{l}$ of blood plus $500 \mu \mathrm{l}$ of $20 \mathrm{mM}$ EDTA (plasma and PBMCs) via retro-orbital bleeding.

75| Perform cervical dislocation.

76| Collect mouse tissues (spleen, lymph nodes, liver and others) immediately. For DNA/protein isolation, freeze tissues in liquid nitrogen or on dry ice. For RNA isolation, place pieces of various tissues in RNA stabilization solution (RNAlater). For cell isolation, place tissues in IMDM medium and keep them on ice. For histology and immunohistochemistry, fix the tissue in formalin and process as paraffin-embedded slides. Examine human hepatocyte repopulation in chimeric liver tissue at the point of euthanasia by staining formalin-fixed, paraffin-embedded slides with human-specific albumin antibody or humanspecific hepatocyte (HepPar1 antibody). Furthermore, human hepatocyte differentiation can be examined using real-time gene expression analysis of liver RNA from humanized and control mice.

77| if you wish to study HCV infection and viral dynamics, examine the viral load by measuring HCV genomic RNA in the serum and liver using established quantitative RT-PCR methods ${ }^{20}$.

78| if you wish to study human immune response, isolate leukocytes from the spleen, liver and lymph nodes using standard leukocyte isolation protocols. Determine the frequency, activation state and number of various human immune cells in the various tissues using standard flow cytometry protocols. Examine anti-HCV $\mathrm{T}$ cell immune response by measuring $\mathrm{T}$ cell activation and/or expansion after antigen stimulation using standard $\mathrm{T}$ cell assays.

79| If you wish to study liver immunopathogenesis and fibrosis, examine hepatitis by immunohistochemistry (IHC); stain paraffin-embedded, formalin-fixed liver slides with antibodies against human immune cells (human $\mathrm{CD} 45^{+}$, human $\mathrm{CD}^{+}$, human $\mathrm{CD}^{+} 8^{+}$and so on) ${ }^{11}$. Examine liver damage by measuring ALT levels in the serum/plasma. Examine liver fibrosis by staining the liver with either Sirius Red/Fast Green or Masson's trichrome stain. Examine human hepatic stellate cell activation by staining with human-specific a-SMA and human-specific GFAP antibodies. In addition, human fibrotic genes expression levels in chimera liver tissue can be examined using human-specific primers (tissue inhibitor of metalloproteinases 1, collagen 1A1) ${ }^{11}$.

\section{? TROUBLESHOOTING}

Troubleshooting advice can be found in Table 1.

\section{- TIMING}

Steps 1-12, liver digestion: $1 \mathrm{~h}$ 
Steps 13-18, liver and HPCs separation: $1 \mathrm{~h}$

Steps 19-24, lymphocyte purification: $1 \mathrm{~h}$

Steps 25-48, CD34+ cell purification: $2 \mathrm{~h}$

Steps 49-51, cell preparation for injection: 30-40 min

Steps 52-63, animal preparation and transplantation: $1.5 \mathrm{~h}$

Steps 64-70, human liver and immune system reconstitution: 3 months

Steps 71 and 72, virus inoculation: $2 \mathrm{~h}$

Steps 73-79, hepatitis virus infection, immune response and liver disease: $2-5$ months

\section{ANTICIPATED RESULTS}

\section{Human liver progenitor cell and CD34+ HSC isolation}

Approximately 5 to $10 \times 10^{6}$ viable liver progenitor cells and 10 to $20 \times 10^{6}$ viable CD34 ${ }^{+}$ HSCs at $80 \%$ purity should be recovered from each fetal liver (15-18 weeks gestation period).

\section{Human liver and immune system reconstitution}

At 16 weeks after transplantation, human immune reconstitution ranges from 10 to $90 \%$, with a mean of $\sim 40 \%$; various human leukocyte lineages are developed in the liver and lymphoid organs. IHC staining with human-specific albumin or HepPar1 antibody shows $\sim 20 \%$ human hepatocytes of total hepatocytes, with reconstitution generally ranging from 15 to $25 \%{ }^{11}$. Human serum albumin levels of humanized mice are rather low at $\sim 200 \mathrm{ng} \mathrm{ml}^{-1}$. Analysis of human hepatocyte metabolism genes using human-specific primers should show elevated gene expression levels ${ }^{11}$.

\section{HCV infection, human immune response and liver disease}

Approximately $50 \%$ of $\mathrm{HCV}$-inoculated mice had detectable HCV genome in the liver; however, serum HCV genome was rarely detected in infected animals. HCV-infected humanized mice should have hepatitis (human immune cell infiltration and cytokine induction) and fibrosis (elevated Sirius red collagen levels, hepatic stellate cell activation) ${ }^{11}$. Note that 8 weeks after inoculation was the earliest analysis and detection time point that showed high incidence of infection, hepatitis and liver fibrosis ${ }^{11}$. Human T cells from HCVinfected humanized mice should show expansion in response to antigen stimulation ${ }^{11}$.

\section{Acknowledgments}

We thank the Su laboratory members for discussion and support. This work was supported in part by grants from a UNC University Cancer Research Fund innovation grant, from the US National Institutes of Health (AI076142, AA018009 to L.S.) and from a UNC Lineberger Comprehensive Cancer Center Postdoctoral Training grant (M.T.B).

\section{References}

1. Mercer DF, et al. Hepatitis C virus replication in mice with chimeric human livers. Nat Med. 2001; 7:927-933. [PubMed: 11479625]

2. Shultz LD, Ishikawa F, Greiner DL. Humanized mice in translational biomedical research. Nat Rev Immunol. 2007; 7:118-130. [PubMed: 17259968]

3. Willinger T, Rongvaux A, Strowig T, Manz MG, Flavell RA. Improving human hemato-lymphoidsystem mice by cytokine knock-in gene replacement. Trends Immunol. 2011; 32:321-327.

[PubMed: 21697012] 
4. Meuleman P, Leroux-Roels G. The human liver-uPA-SCID mouse: a model for the evaluation of antiviral compounds against HBV and HCV. Antiviral Res. 2008; 80:231-238. [PubMed: 18706933]

5. Meuleman P, et al. Morphological and biochemical characterization of a human liver in a uPASCID mouse chimera. Hepatology. 2005; 41:847-856. [PubMed: 15791625]

6. Azuma H, et al. Robust expansion of human hepatocytes in $\mathrm{Fah}^{-/-} / \mathrm{Rag}^{-/} / \mathrm{Il} 2 \mathrm{rg}^{-/-}$mice. Nat Biotechnol. 2007; 25:903-910. [PubMed: 17664939]

7. Bissig KD, Le TT, Woods NB, Verma IM. Repopulation of adult and neonatal mice with human hepatocytes: a chimeric animal model. Proc Natl Acad Sci USA. 2007; 104:20507-20511. [PubMed: 18077355]

8. Bissig KD, et al. Human liver chimeric mice provide a model for hepatitis B and C virus infection and treatment. J Clin Invest. 2010; 120:924-930. [PubMed: 20179355]

9. Traggiai E, et al. Development of a human adaptive immune system in cord blood cell-transplanted mice. Science. 2004; 304:104-107. [PubMed: 15064419]

10. Pajvani UB, et al. Fat apoptosis through targeted activation of caspase 8: a new mouse model of inducible and reversible lipoatrophy. Nat Med. 2005; 11:797-803. [PubMed: 15965483]

11. Washburn ML, et al. A humanized mouse model to study hepatitis $\mathrm{C}$ virus infection, immune response, and liver disease. Gastroenterology. 2011; 140:1334-1344. [PubMed: 21237170]

12. Hayashi H, Sakai T. Animal models for the study of liver fibrosis: new insights from knockout mouse models. Am J Physiol Gastrointest Liver Physiol. 2011; 300:G729-G738. [PubMed: 21350186]

13. Merchante N, et al. Survival and prognostic factors of HIV-infected patients with HCV-related end-stage liver disease. AIDS. 2006; 20:49-57. [PubMed: 16327319]

14. Operskalski EA, Kovacs A. HIV/HCV co-infection: pathogenesis, clinical complications, treatment, and new therapeutic technologies. Curr HIV/AIDS Rep. 2011; 8:12-22. [PubMed: 21221855]

15. de Jong YP, Rice CM, Ploss A. New horizons for studying human hepatotropic infections. J Clin Invest. 2010; 120:650-653. [PubMed: 20179350]

16. Robinet E, Baumert TF. A first step towards a mouse model for hepatitis $\mathrm{C}$ virus infection containing a human immune system. J Hepatol. 2011; 55:718-720. [PubMed: 21616105]

17. Haridass D, et al. Repopulation efficiencies of adult hepatocytes, fetal liver progenitor cells, and embryonic stem cell-derived hepatic cells in albumin-promoter-enhancer urokinase-type plasminogen activator mice. Am J Pathol. 2009; 175:1483-1492. [PubMed: 19717639]

18. Mallet VO, et al. Conditional cell ablation by tight control of caspase- 3 dimerization in transgenic mice. Nat Biotechnol. 2002; 20:1234-1239. [PubMed: 12434157]

19. Zhang L, Kovalev GI, Su L. HIV-1 infection and pathogenesis in a novel humanized mouse model. Blood. 2007; 109:2978-2981. [PubMed: 17132723]

20. Lindenbach BD, et al. Cell culture-grown hepatitis $\mathrm{C}$ virus is infectious in vivo and can be recultured in vitro. Proc Natl Acad Sci USA. 2006; 103:3805-3809. [PubMed: 16484368] 


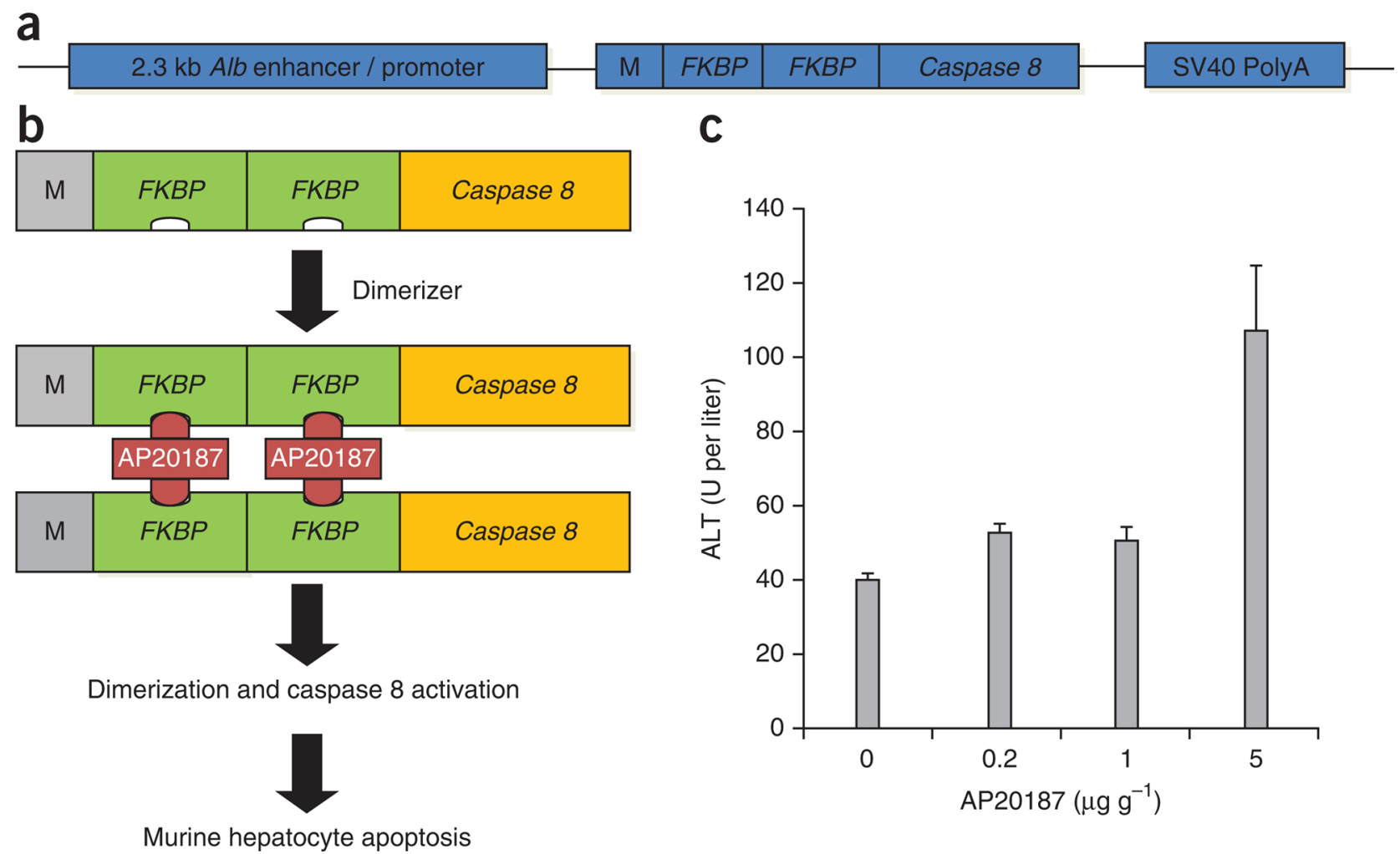

AP20187 treatment induces temporal liver-specific damage in AFC8 mice. (a) The structure of the AFC8 mouse model transgene construct. (b) Activation of the AFC8 model's transgene with the dimerizer AP20187 induces apoptosis through Caspase 8 signaling. (c) AFC8 mice (4-6 weeks old) were injected with AP20187 at indicated doses in accordance with the protocols and guidelines approved by the UNC Institutional Animal Care and Use Committee (IACUC); ALT was measured in serum at $1 \mathrm{~d}$ after drug treatment. Two animals per group $(n=2)$; error bars show means \pm s.d. 


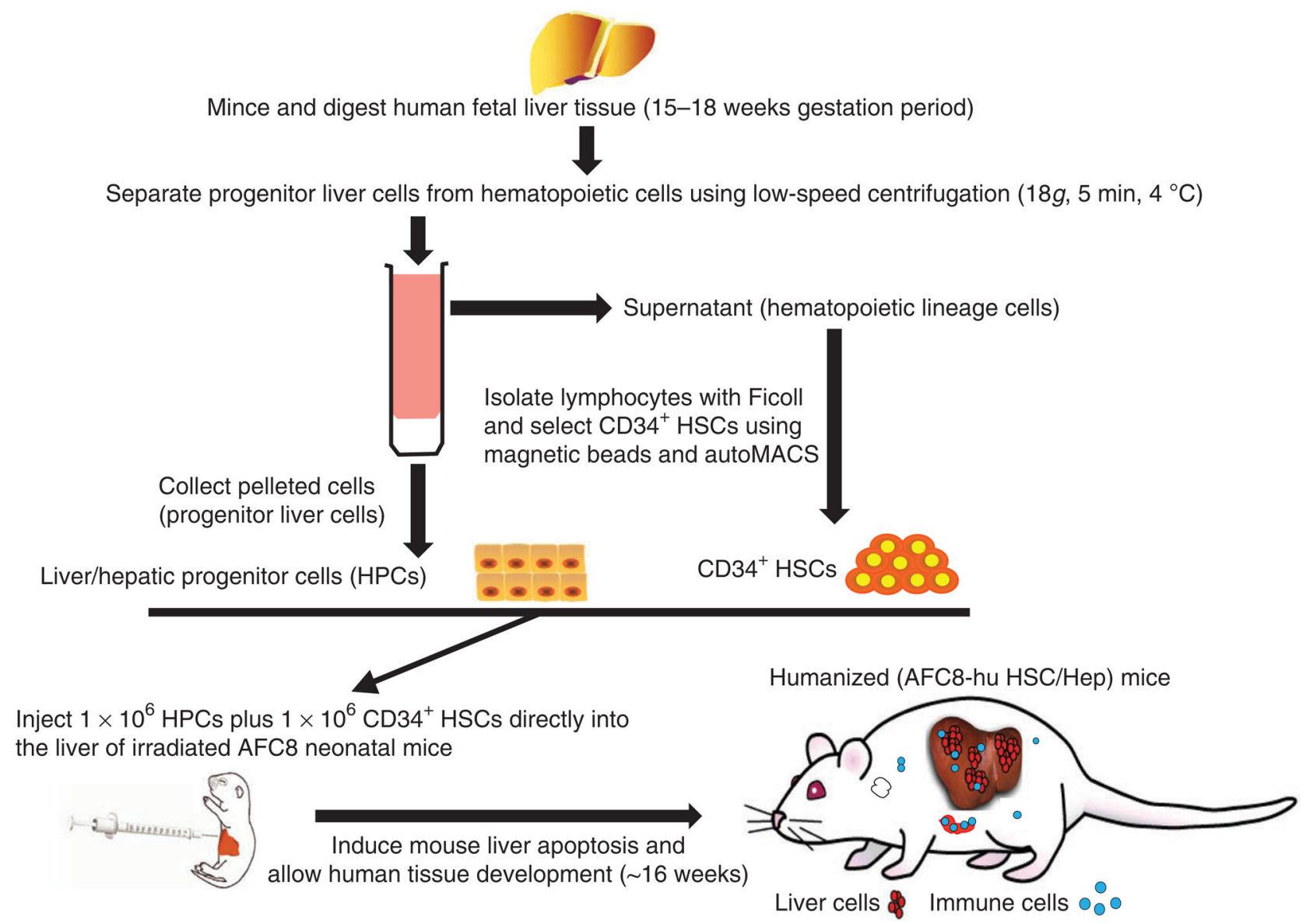

Figure 2.

Procedure overview: humanization of the liver and immune system in the AFC8 mice. Overview of hematopoietic stem cell and liver progenitor cell isolation from human fetal liver tissue and construction of the AFC8-hu HSC/Hep mouse model. 
a

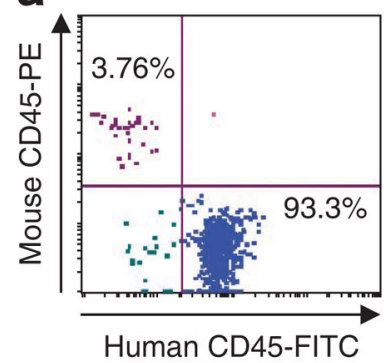

b

$\%$ Human immune reconstitution : $=$ Human $\mathrm{CD} 45 \% \div$ total $\mathrm{CD} 45 \%$

$=$ (Lower right quadrant) $\div$ (upper left + lower right quadrants)

$=(93.3 \%) \div(3.76 \%+93.3 \%)=0.96$

$96 \%$ human immune reconstitution

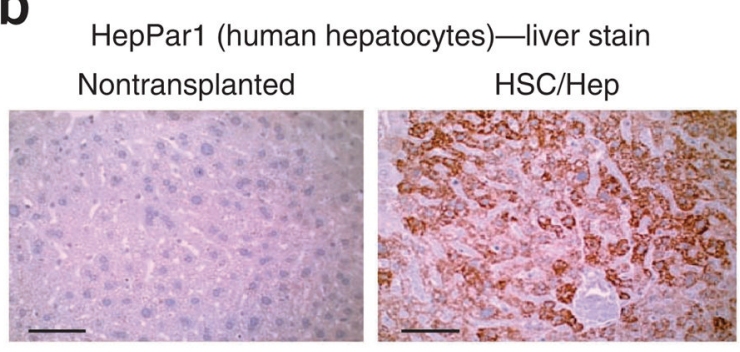

Figure 3.

Human immune system and liver reconstitution in AFC8-hu mice. (a) PBMCs from humanized mice were stained with 7-AAD (live/dead cell marker) and human and mouse CD45, and the percentage of human immune cell reconstitution in the chimeric mouse was determined. (b) HepPar1 (human hepatocyte)-stained liver tissues from nontransplanted and AFC8 mice transplanted with fetal liver-derived cells. Scale bars, $50 \mu \mathrm{m}$. 


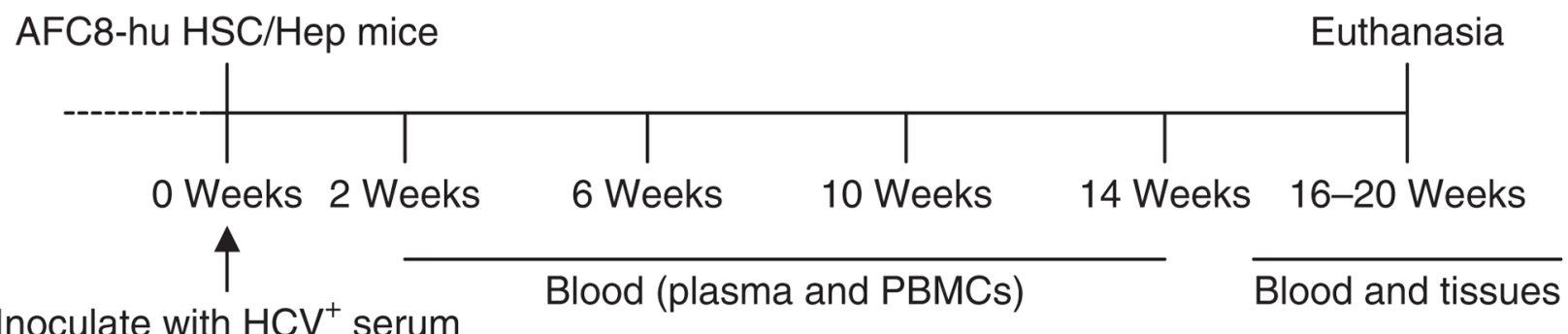

Figure 4.

Procedure overview: HCV infection and immunopathogenesis. Analysis in the AFC8-hu mouse model. Timeline for HCV infection, immune response and liver disease analysis in the AFC8-hu HSC/Hep mouse model. Blood is collected via tail bleeding for longitudinal analysis of plasma and PBMCs. Blood and tissues (liver, spleen, lymph nodes, bone marrow and other internal organs) are collected for analysis at approximately 16-20 weeks after inoculation. 
TABLE 1

Troubleshooting table.

\begin{tabular}{lll}
\hline Step & Problem & Solution \\
\hline 17 & $\begin{array}{l}\text { Removal of red blood cells from the liver progenitor cell } \\
\text { fraction }\end{array}$ & $\begin{array}{l}\text { If the pellet is red, repeat Steps 15 and 16 until the pellet is yellow or } \\
\text { white }\end{array}$ \\
69,70 & $\begin{array}{l}\text { Infection of animals with various pathogens, including } \\
\text { bacteria, will result in very low human reconstitution } \\
\text { and illness }\end{array}$ & $\begin{array}{l}\text { Maintain a clean facility and a germ-free colony through sterile } \\
\text { procedures and techniques and the administration of bacteriocide and } \\
\text { bacteriostatic agents }\end{array}$ \\
\hline
\end{tabular}

